\title{
Wenxiang: a web-server for drawing wenxiang diagrams
}

\author{
Kuo-Chen Chou $^{1^{*}}$, Wei-Zhong Lin $^{2,3}$, Xuan Xiao ${ }^{1,3}$ \\ ${ }^{1}$ Gordon Life Science Institute, San Diego, California, USA; ${ }^{*}$ Corresponding Author: kcchou@gordonlifescience.org \\ ${ }^{2}$ Information Science and Technology School, Donghua University, Shanghai, China; \\ ${ }^{3}$ Computer Department, Jingdezhen Ceramic Institute, Jingdezhen, China.
}

Received 15 September 2011, revised 16 October 2011; accepted 23 October 2011.

\begin{abstract}
The wenxiang diagram was proposed to represent $\alpha$-helices in a 2D (two dimensional) space (Chou, K.C., Zhang, C.T., Maggiora, G.M. Proteins: Struct., Funct., Genet., 1997, 28, 99-108). It has the capacity to provide more information in a 2D plane about each of the constituent amino acid residues in an $\alpha$-helix, and is particularly useful for studying and analyzing amphiphilic helices. To meet the increasing requests for getting the program of generating wenxiang diagrams, a user-friendly web-server called "Wenxiang" has been established. It is accessible to the public at the web-site http://www.jci-bioinfo.cn/wenxiang2 or http://icpr.jci.edu.cn/bioinfo/wenxiang2. Furthermore, for the convenience of users, here we provide a step-to-step guide for how to use the Wenxiang web-server to generate the desired wenxiang diagrams.
\end{abstract}

Keywords: Amphiphilic Helix; Helix-Helix Interaction; Hydrophobic; Hydrophilic; 2D Diagram; Wenxiang Diagram; Helical Wheel Diagram

\section{INTRODUCTION}

The $\alpha$-helix is one of the most basic and fundamental elements in protein structure [1]. Owing to their regularity, $\alpha$-helices have had an immense influence on our understanding of protein structure. Also, owing to their elasticity, many marvelous biological functions of proteins can be revealed through the low-frequency accordion-like motions of $\alpha$-helices (see, e.g., [2-4] and a comprehensive review [5]).

Using graphical or diagrammatic approaches to study biological systems can provide an intuitive picture or useful insights for helping analyzing their complicated mechanisms, as demonstrated by many studies on a series of important biological topics, such as enzymecatalyzed reactions [6-9], protein folding kinetics [10,11], inhibition of HIV-1 reverse transcriptase [12-14], inhibition kinetics of processive nucleic acid polymerases and nucleases [15], drug metabolism systems [16], protein attribute prediction $[17,18]$, protein sequence evolution [19], among many others.

Since the "pen-and-paper space" and most of the "publication space" are two-dimensional (2D) space, it is desirable to represent the 3D structures of $\alpha$-helices on a 2D plane. In this regard, two different kinds of diagrams are usually used to represent $\alpha$-helices. One is called the "helical wheel diagram" [20], and the other called "wenxiang diagram" [21]. The name of the latter came from the fact that it looks like a coil-like incense used in China to repel mosquitoes, i.e., Chinese “蚊香” (pronounced as "wenxiang"). For its shape and appearance, click the link http://wapedia.mobi/zh/\%E8\%9A\%8A\%E9\%A6\%99.

According to its original definition [21], a 2D wenxiang diagram is generated by a conical projection of an $\alpha$-helix onto a plane perpendicular to its axis (Figure 1). For a rigorous definition of an $\alpha$-helix's axis, see [22]. In the $2 \mathrm{D}$ wenxiang diagram each amino acid residue is represented by a circle with a letter to indicate its single character code, while its location in the 2D plane is defined by both an angle around the helix's axis and the distance from the center, the intersection point between the 2D plane and the helix's axis, as elaborated in [21].

As a $2 \mathrm{D}$ representation, the wenxiang diagram has the following features: (i) able to show the relative locations of the amino acids in an alpha-helix regardless how long it is; (ii) able to indicate the direction of an alpha-helix; and (iii) having the capacity to provide more information about each of the constituent amino acid residues in an $\alpha$-helix [21].

With these features, the wenxiang diagram can provides an intuitive and easily visualizable picture in a $2 \mathrm{D}$ space that characterizes the disposition of different residue types in an $\alpha$-helix.

As is known, except for transmembrane helices, $\alpha$-helices in globular proteins are generally amphiphilic, i.e., consist of two types of residues, hydrophobic and hydrophilic, with the number of each type being roughly 




Figure 1. Conical projection of a helix $\mathrm{H}$ onto a plane perpendicular to the helix $\mathrm{H}$. The dashed lines represent the radiating lines from the apex A. The image of helix $\mathrm{H}$ on the projection plane is within the ring area $\mathrm{R}$ : the closer the part of helix $\mathrm{H}$ is from the apex $\mathrm{A}$, the further away its image is from the center of the ring $\mathrm{R}$.

equal. Wenxiang diagrams are particularly useful to help gain insights into the interactions among amphiphilic helices or between proteins that contain amphiphilic helices $[23,24]$.

Recently we have received many requests for the program of generating wenxiang diagrams. To address these requests in a unified manner, we have established a webserver by which one can easily generate the wenxiang diagram for any $\alpha$-helix according to its amino acid sequence, as described below.

\section{EQUIPMENT}

You need a computer with access to the Internet and a web browser.

\section{PROCEDURE}

You should follow the guide below to generate the wenxiang diagram for an $\alpha$-helix.

1) Go to the web page at http://www.jci-bioinfo.cn/ wenxiang2 or http://icpr.jci.edu.cn/bioinfo/wenxiang2, and you will see the top page of the Wenxiang web-server on your computer screen, as shown in Figure 2.

2) Click the Read Me button to see the user's instructions, which can be elaborated via the following steps.

3) Either type or copy and paste the $\alpha$-helix amino acid sequence (in single letter code) into the input box at the lower centre of Figure 2. If you are not sure about the input format, click on the button Example right above the input box to see the format of an acceptable input sequence.

4) Click on the Black \& White button to generate a black-and-white wenxiang diagram (Figure 3), where hydrophobic residues are shown by "black-filled circles with white characters" while hydrophilic residues by "open circles with black characters". It will take about 20 seconds before the desired diagram is completely shown on the screen.

5) Or alternatively, click the button Color to generate a color wenxiang diagram (Figure 4), where hydrophobic residues are shown by "red-filled circles with white characters" while hydrophilic residues by "blue-filled circles with black characters.

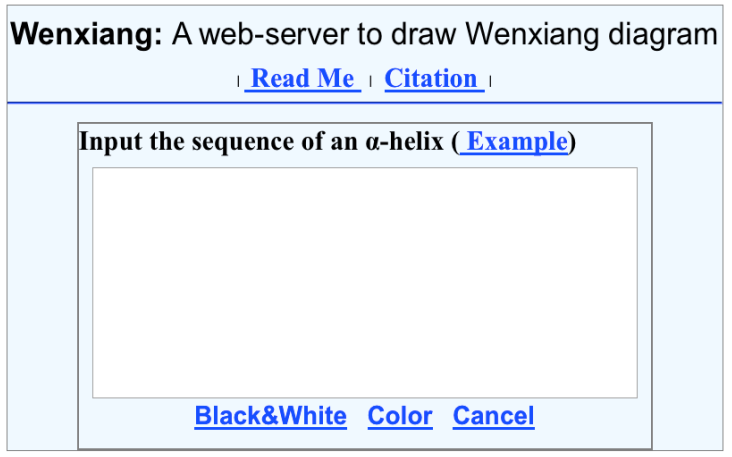

Figure 2. A screenshot of the top page of the Wenxiang web-server at http://www.jci-bioinfo.cn/wenxiang2 or http://icpr.jci.edu.cn/bioinfo/wenxiang2.

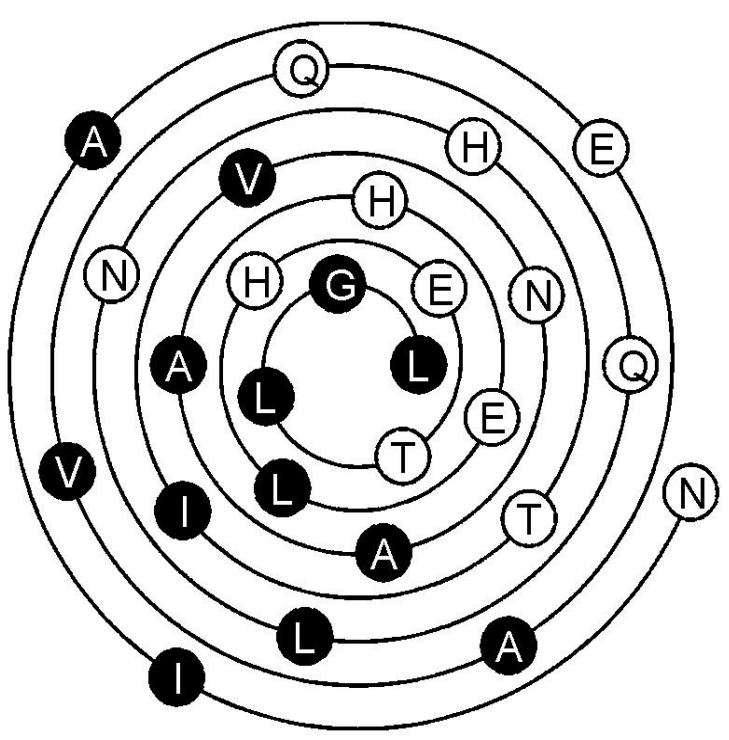

Figure 3. The black-and-white wenxiang diagram generated by clicking the Black \& White button of the web-server for the input sequence taken from the Example; the "black-filled circles with white characters" represent the hydrophobic residues, while the "open circles with black characters" hydrophilic residues. 


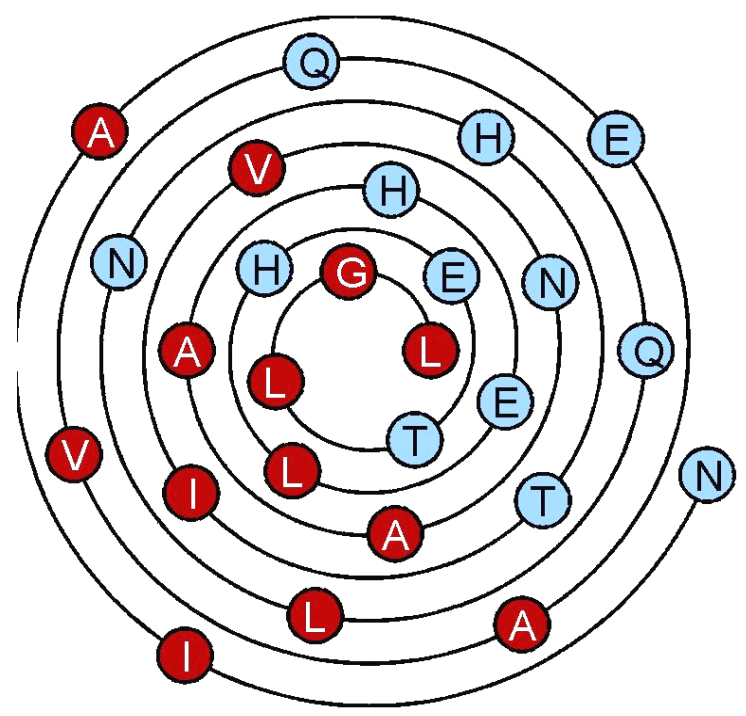

Figure 4. The color wenxiang diagram generated by clicking the Color button of the web-server for the same input sequence as in Figure 3. The "red-filled circles with white characters" represent hydrophobic residues, while the "blue-filled circles with black characters" represent the hydrophilic residues.

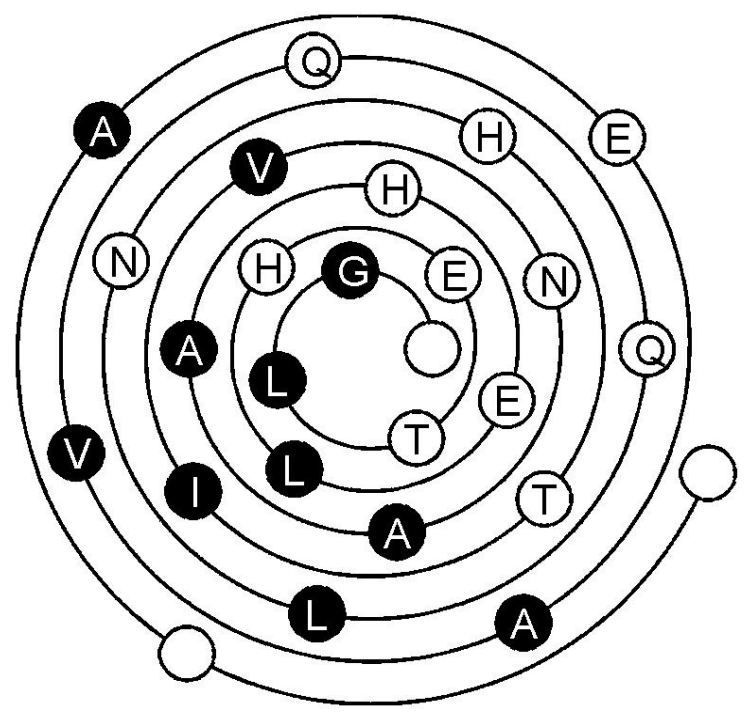

Figure 5. The black-and-white wenxiang diagram generated by clicking the Black \& White button of the web-server for the input sequence XXAEAVQQLNHTIVNAAHELHETLGX that contains invalid codes in its $1^{\text {st }}, 2^{\text {nd }}$ and last positions. The three "open circles" represent the three invalid codes at the $1^{\text {st }}, 2^{\text {nd }}$, and last positions, respectively.

6) To save the wenxiang diagram, click it with the right mouse button.

7) Click on the Citation button to find the relevant papers that document the detailed development of wenxiang diagram and some of its applications.

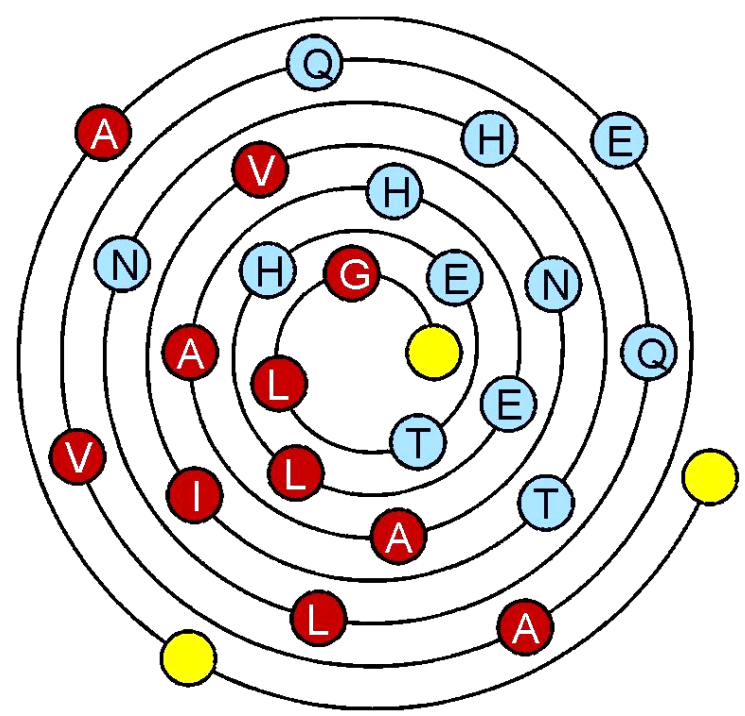

Figure 6. The color wenxiang diagram generated by clicking the Color button of the web-server for the same input sequence in Figure 5. The three "yellow-filled circles" represent the three invalid codes at the $1^{\text {st }}, 2^{\text {nd }}$, and last positions of the sequence, respectively.

\section{DISCUSSION}

The valid codes for a native $\alpha$-helix should be within the 20 characters ACDEFGHIKLMNPQRSTVWY, which represent 20 native amino acids, respectively.

If your sequence contains some invalid codes, after clicking the Black \& White button, a warning message will occur on the screen. If you still want to generate the wenxiang diagram, just click the $\mathbf{O K}$ button. However, the wenxiang diagram thus generated will contain some "open circles" with each corresponding to one of the invalid codes. For example, for the input sequence XXAEAVQQLNHTIVNAAHELHETLGX where the first two codes and the last one are invalid, the wenxiang diagram thus generated will look like the one shown in Figure 5.

The similar situation will also occur when clicking the Color button to generate the color wenxiang diagram for the above invalid sequence, in which case each of the invalid codes will be shown by a "yellow-filled circle" as shown in Figure 6.

The wenxiang diagrams thus generated for non-native helices that contain invalid codes may give you some flexibility for studying hypothetical $\alpha$-helices.

\section{ACKNOWLEDGEMENTS}

This work was supported by the grants from the National Natural Science Foundation of China (No. 60961003), the Key Project of Chinese Ministry of Education (No. 210116), and the Department of Education of Jiangxi Province (No. GJJ11557). 


\section{REFERENCES}

[1] Richardson, J.S. (1981) The anatomy and taxonomy of protein structure. Advances in Protein Chemistry, 34, 167-339. doi:10.1016/S0065-3233(08)60520-3

[2] Chou, K.C. (1983) Identification of low-frequency modes in protein molecules. Biochemical Journal, 215, 465-469. PMID 6362659

[3] Chou, K.C. (1989) Low-frequency resonance and cooperativity of hemoglobin. Trends in Biochemical Sciences, 14, 212. doi:10.1016/0968-0004(89)90026-1

[4] Martel, P. (1992) Biophysical aspects of neutron scattering from vibrational modes of proteins. Progress in Biophysics and Molecular Biology, 57, 129-179. doi:10.1016/0079-6107(92)90023-Y

[5] Chou, K.C. (1988) Review: Low-frequency collective motion in biomacromolecules and its biological functions. Biophysical Chemistry, 30, 3-48. doi:10.1016/0301-4622(88)85002-6

[6] Chou, K.C. and Forsen, S. (1980) Graphical rules for enzyme-catalyzed rate laws. Biochemical Journal, 187, 829-835. PMID 7188428

[7] Zhou, G.P. and Deng, M.H. (1984) An extension of Chou's graphical rules for deriving enzyme kinetic equations to system involving parallel reaction pathways. Biochemical Journal, 222, 169-176. PMID 6477507

[8] Chou, K.C. (1989) Graphic rules in steady and nonsteady enzyme kinetics. Journal of Biological Chemistry, 264, 12074-12079. PMID 2745429

[9] Andraos, J. (2008) Kinetic plasticity and the determination of product ratios for kinetic schemes leading to multiple products without rate laws: New methods based on directed graphs. Canadian Journal of Chemistry, 86, 342-357. doi: $10.1139 / \mathrm{v} 08-020$

[10] Chou, K.C. (1990) Review: Applications of graph theory to enzyme kinetics and protein folding kinetics. Steady and non-steady state systems. Biophysical Chemistry, 35, 1-24. doi:10.1016/0301-4622(90)80056-D

[11] Shen, H.B., Song, J.N. and Chou, K.C. (2009) Prediction of protein folding rates from primary sequence by fusing multiple sequential features. Journal of Biomedical Science and Engineering (JBiSE), 2, 136-143 (openly accessible at http://www.scirp.org/journal/jbise). doi: $10.4236 /$ jbise.2009.23024

[12] Althaus, I.W., Chou, J.J., Gonzales, A.J., Diebel, M.R., Chou, K.C., Kezdy, F.J., Romero, D.L., Aristoff, P.A., Tarpley, W.G. and Reusser, F. (1993) Steady-state kinetic studies with the non-nucleoside HIV-1 reverse transcriptase inhibitor U-87201E. Journal of Biological Chemistry, 268, 6119-6124. PMID 7681060

[13] Althaus, I.W., Gonzales, A.J., Chou, J.J., Diebel, M.R., Chou, K.C., Kezdy, F.J., Romero, D.L., Aristoff, P.A., Tarpley, W.G. and Reusser, F. (1993) The quinoline U-78036 is a potent inhibitor of HIV-1 reverse transcriptase. Journal of Biological Chemistry, 268, 14875-14880.

\section{PMID 7686907}

[14] Althaus, I.W., Chou, J.J., Gonzales, A.J., Diebel, M.R., Chou, K.C., Kezdy, F.J., Romero, D.L., Aristoff, P.A., Tarpley, W.G. and Reusser, F. (1993) Kinetic studies with the nonnucleoside HIV-1 reverse transcriptase inhibitor U-88204E. Biochemistry, 32, 6548-6554. doi:10.1021/bi00077a008

[15] Chou, K.C., Kezdy, F.J. and Reusser, F. (1994) Review: Steady-state inhibition kinetics of processive nucleic acid polymerases and nucleases. Analytical Biochemistry, 221, 217-230. doi:10.1006/abio.1994.1405

[16] Chou, K.C. (2010) Graphic rule for drug metabolism systems. Current Drug Metabolism, 11, 369-378. doi:10.2174/138920010791514261

[17] Xiao, X., Wang, P. and Chou, K.C. (2008) Predicting protein structural classes with pseudo amino acid composition: An approach using geometric moments of cellular automaton image. Journal of Theoretical Biology, 254, 691-696. doi:10.1016/j.jtbi.2008.06.016

[18] Xiao, X., Wang, P. and Chou, K.C. (2009) GPCR-CA: A cellular automaton image approach for predicting Gprotein-coupled receptor functional classes. Journal of Computational Chemistry, 30, 1414-1423. doi: $10.1002 /$ icc. 21163

[19] Wu, Z.C., Xiao, X. and Chou, K.C. (2010) 2D-MH: A web-server for generating graphic representation of protein sequences based on the physicochemical properties of their constituent amino acids. Journal of Theoretical Biology, 267, 29-34. doi:10.1016/j.jtbi.2010.08.007

[20] Schiffer, M. and Edmundson, A.B. (1967) Use of helical wheels to represent the structures of proteins and to identify segments with helical potential. Biophysical Journal, 7, 121-135. doi:10.1016/S0006-3495(67)86579-2

[21] Chou, K.C., Zhang, C.T. and Maggiora, G.M. (1997) Disposition of amphiphilic helices in heteropolar environments. Proteins: Structure, Function, and Genetics, 28, 99-108.

doi:10.1002/(SICI)1097-0134(199705)28:1<99::AID-PR OT10>3.0.CO;2-C

[22] Chou, K.C., Nemethy, G. and Scheraga, H.A. (1984) Energetic approach to packing of a-helices: 2. General treatment of nonequivalent and nonregular helices. Journal of American Chemical Society, 106, 3161-3170. doi:10.1021/ja00323a017

[23] Zhou, G.P. (2011) The disposition of the LZCC protein residues in wenxiang diagram provides new insights into the protein-protein interaction mechanism. Journal of Theoretical Biology, 284, 142-148. doi:10.1016/j.jtbi.2011.06.006

[24] Zhou, G.P. (2011) The structural determinations of the leucine zipper coiled-coil domains of the cGMP-Dependent protein kinase I alpha and its interaction with the myosin binding subunit of the myosin light chains phosphase. Proteins \& Peptide Letters, 18, 966-978. $\underline{\text { PMID } 21592084}$ 